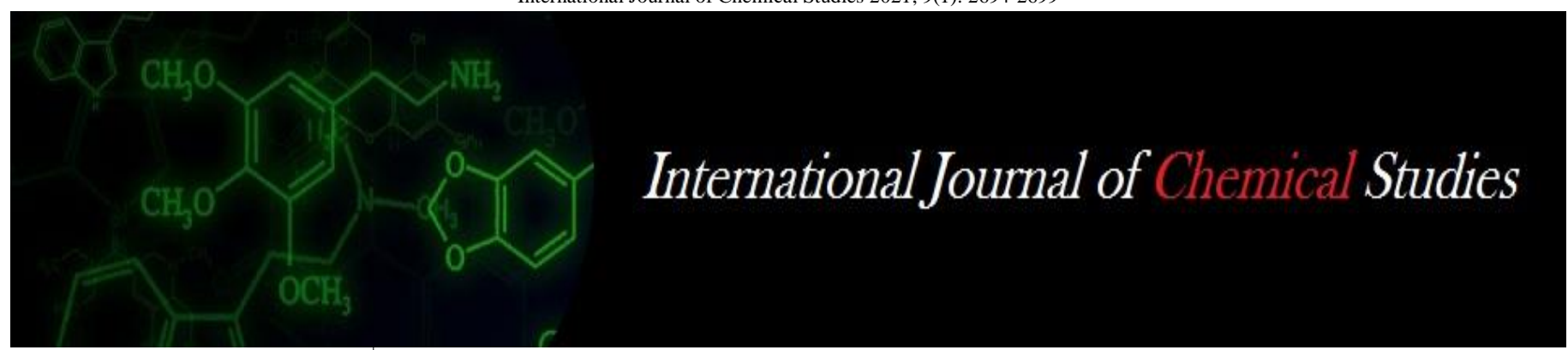

P-ISSN: 2349-8528

E-ISSN: 2321-4902

www.chemijournal.com

IJCS 2021; 9(1): 2694-2699

(C) 2021 IJCS

Received: 11-10-2020

Accepted: 21-11-2020

Pooja Upadhyay

Centre of Advanced Studies,

Department of Plant Pathology,

College of agriculture, GBPUA \&

T Pantnagar, U.S. Nagar,

Uttarakhand, India

\section{AK Tewari}

Centre of Advanced Studies,

Department of Plant Pathology,

College of agriculture, GBPUA \&

T Pantnagar, U.S. Nagar,

Uttarakhand, India

\section{Devanshu Dev}

Centre of Advanced Studies, Department of Plant Pathology, College of agriculture, GBPUA \& T Pantnagar, U.S. Nagar,

Uttarakhand, India

GR Daniel

Centre of Advanced Studies, Department of Plant Pathology, College of agriculture, GBPUA \& T Pantnagar, U.S. Nagar,

Uttarakhand, India
Corresponding Author: Pooja Upadhyay Centre of Advanced Studies, Department of Plant Pathology, College of agriculture, GBPUA \& T Pantnagar, U.S. Nagar, Uttarakhand, India

\section{Evaluation of different Brassica genotypes under field conditions against white rust disease (Albugo candida) of rapeseed-mustard for resistant sources}

\author{
Pooja Upadhyay, AK Tewari, Devanshu Dev and GR Daniel
}

DOI: $\underline{\text { htps://doi.org/10.22271/chemi.2021.v9.i1al.11632 }}$

\begin{abstract}
White rust or white blister disease caused by oomycete fungi, Albugo candia is one of the major devastating disease of rapeseed mustard. Continuous emergence of new races of the pathogen is responsible for breaking down of the resistance of already existing resistant cultivars. So there is need for the evaluation of existing resistant cultivars as well as new Brassica cultivars to find out stable resistance sources against the disease. In this context, 82 different Brassica genotypes, including 10 different Brassica species were evaluated in field, under natural conditions for the confirmation of resistance sources against Albugo candida in two successive years 2017-18 and 2018-19. Observations of percent disease severity and phenotypic disease reaction were taken at different time intervals of 70, 80 and 90 DAS. Infection rate and area under disease progress curve (AUDPC) were calculated for each genotype. Out of 82 Brassica genotypes evaluated, 26 were found free from the disease and 28 genotypes showed moderately resistant reaction while rest of the genotypes showed susceptible reaction at 90DAS. Minimum infection rate (0.0949) and AUDPC (69.44) was observed in DRMRJ 127 and SIVT-17-19 while maximum infection rate (0.1008) and AUDPC (588.0) was observed in $71 \mathrm{~J} 0002$ and RH 30, respectively at 90 DAS.
\end{abstract}

Keywords: Albugo candida, resistant, AUDPC, white rust, Brassica genotypes

\section{Introduction}

Oilseed Brassicas are important oilseed crops, grown as fodder, source of oil, condiments and vegetables around the world with global production of 584.3 million tonnes $(\mathrm{mt})$ in 2019-20 (FAO, 2020). In India, oilseed crops comprise $26.20 \mathrm{mha}$, area with production of $35.35 \mathrm{mt}$ and yield of $1328 \mathrm{~kg} / \mathrm{ha}$. Oilseed Brassica includes eight different species namely toria, yellow Sarson, Indian mustard, gobhi Sarson, Taramira, Brown Sarson and Karan Rai. Among them rapeseed-mustard attain prime position with total area of $6.64 \mathrm{mha}$, total production $8.1 \mathrm{mt}$ and productivity $1980 \mathrm{~kg} / \mathrm{ha}$ (Anonymous, 2020) ${ }^{[1]}$. Rapeseed-mustard is susceptible to a number of diseases eg. Alternaria blight, White rust, Sclerotinia stem rot and Downey mildew which limit its production. Among them, white rust disease caused by Albugo candida (Pers. ex Lev.) Kuntze, is wide spread and highly destructive disease in many agronomically important Brassica species as pathogen can spread systemically and cause severe malformation of the inflorescence through hypertrophy and hyperplasia resulting in staghead formation (Punjabi et al., 2010) ${ }^{[16]}$. The disease causes annual yield loss of 20-60 percent in rapeseed mustard crop (Saharan et al., 1984, Saharan and Verma, 1992, Bisht et al. 1994 and Kolte, 1996, Kalpana et al, 2017) ${ }^{[20,17,3,8]}$. Protectant fungicides have been recommended for controlling white rust disease in rapeseed-mustard (Kolte and Tewari., 1980) ${ }^{[9]}$ and Kolte and Awasthi, 1981, Kalpana et al, 2019) ${ }^{[10,7]}$. But due to concern of environmental hazards, high cost of chemicals and problem of non-uniform spraying due to plant height, farmers are usually reluctant to use these fungicides for the management of the disease. At present best alternative method for management of this disease is identification of resistant cultivars which is also most ecofriendly and cost effective disease management strategy for this disease. Availability of resistant source has been reported by several workers and different criteria have been used by them to determine the relative resistance of various genotypes in oilseed Brassica 
(Ashrufuzzaman, et al., 1996, Kumar and Kolte., 2001) [2, 11]. However, less work has been done to understand the various aspect of disease component in resistant and susceptible genotypes. In this view, it is very important to identify and confirm the resistant sources. So that some promising and potential genotypes could be identified and used as donor sources in resistant breeding programme. Due to variability and wide distribution of white rust pathogen, new races of Albugo candida pathogen emerge which challenge the resistance of existing cultivars. Hence, along with screening of new Brassica genotypes, continuous evaluation of already reported resistant cultivars is also needed for finding stable resistant sources against the pathogen. Considering the problems, the present investigation was undertaken for the confirmation of resistant sources in Brassica genotypes against $A$. candida.

\section{Material and Method}

Brassica genotypes (82 no.) including 10 different Brassica species were evaluated against $A$. candida pathogen in field under natural condition during the crop season 2017-18 and 2018-19 in Crop research centre, GBPUA\&T, Pantnagar. Following Brassica genotypes (Table 1) were used for evaluation against white rust disease.

Table 1: Brassica genotypes for evaluation against $A$. candida under field condition

\begin{tabular}{|c|c|c|c|c|c|c|c|c|}
\hline Si. No. & Genotypes & Species & Si no. & Genotype & Species & Si no. & Genotype & Species \\
\hline 1 & Varuna & B. juncea & 29 & NBPGR 407 & - & 57 & DRMRIJ-12-06 & B. juncea \\
\hline 2 & Pusa Bold & B. juncea & 30 & NBPGR 410 & - & 58 & NPJ 217 & B. juncea \\
\hline 3 & TL 15 & B. rapa var.toria & 31 & NBPGR 426 & - & 59 & EC 399301 & B. juncea \\
\hline 4 & Candle & B. rapa var. Brown sarson & 32 & NBPGR 427 & - & 60 & RMM-09-05-01 & B. juncea \\
\hline 5 & BSH 1 & B. rapa var. Brown sarson & 33 & NBPGR 428 & - & 61 & DRMRJ 127 & B. juncea \\
\hline 6 & Kranti & B. juncea & 34 & NBPGR 429 & - & 62 & RMWR-09-05-01 & B. juncea \\
\hline 7 & Ragini & B. rapa & 35 & NBPGR 433 & - & 63 & RH.14.001 & B. juncea \\
\hline 8 & RL 1359 & B. juncea & 36 & NBPGR 434 & - & 64 & DRMR 2035 & B. juncea \\
\hline 9 & Pusa Kalyani & B. rapa var. Brown sarson & 37 & NBPGR 437 & - & 65 & DRMR 2019 & B. juncea \\
\hline 10 & Torch & B. rapa var. Brown sarson & 38 & NBPGR 439 & - & 66 & RLC-5 & B. juncea \\
\hline 11 & Tobin & B. rapa var. Brown sarson & 39 & PRD.14.16 & - & 67 & DRMR 12.39 & B. juncea \\
\hline 12 & Bhawani & B. rapa var.toria & 40 & PRD.14.25 & - & 68 & DRMRIJ 12.40 & B. juncea \\
\hline 13 & Sinapis alba & S. alba & 41 & PRD.34.20 & - & 69 & RL-JEB-52 & B. juncea \\
\hline 14 & DLSC-1 & B. carinata & 42 & PAB.14.57 & - & 70 & NPJ 217 & B. juncea \\
\hline 15 & YSPB 24 & B. rapa var. yellow sarson & 43 & IC 413293 & - & 71 & DRMR-12-48 & B. juncea \\
\hline 16 & Wester & B. napus & 44 & IC 426528 & - & 72 & RMM-09-6-1 & B. juncea \\
\hline 17 & Heera & B. juncea & 45 & IC 597932 & - & 73 & PDZ 5 & B. juncea \\
\hline 18 & Cutlass & B. juncea & 46 & IC 3175280 & - & 74 & ABS(3)-15 & B. juncea \\
\hline 19 & Sangam & B. nigra & 47 & IC 313378 & - & 75 & RTM-10-9-1 & B. juncea \\
\hline 20 & RH 30 & B. juncea & 48 & IC 313379 & - & 76 & PDZ 3 & B. juncea \\
\hline 21 & NRCDR 515 & B. juncea & 49 & IC 265495 & - & 77 & DRMR 5206 & B. juncea \\
\hline 22 & Eureca sativa & E. sativa & 50 & IC 298024 & - & 78 & $71 \mathrm{~J} 0002$ & B. juncea \\
\hline 23 & Donskaja & B. juncea & 51 & IC 597932 & - & 79 & RTM 16.24 & B. carinata \\
\hline 24 & EC 41329 & B. juncea & 52 & RMWR-09-05 & B. juncea & 80 & SIVT 17.108 & B. carinata \\
\hline 25 & NBPGR 1 & - & 53 & DRMRIJ-12-65 & B. juncea & 81 & SIVT 17.19 & B. juncea \\
\hline 26 & NBPGR 8 & - & 54 & DRMRIJ-12-43 & B. juncea & 82 & SBZ 1330 & B. juncea \\
\hline 27 & NBPGR 15 & - & 55 & DRMRIJ-12-02 & B. juncea & & & \\
\hline 28 & NBPGR 352 & - & 56 & RH 1231 & B. juncea & & & \\
\hline
\end{tabular}

\subsection{Evaluation under natural field conditions}

The Brassica genotypes were sown on Oct.15, 2017 in a Randomized Block Design. Two rows of each with $3 \mathrm{~m}$ length were sown with plant to plant distance of $5-10 \mathrm{~cm}$ with a susceptible check (Varuna) sown after each two rows. Thinning of plants was done after 15 days of germination and two irrigations were applied during entire crop growth. Recommended doses of $\mathrm{N}_{80} \mathrm{P}_{40} \mathrm{~K}_{40}$ sulphur $40 \mathrm{Kg} / \mathrm{ha}$ was applied in the field. Half dose of nitrozen in the form of Urea was applied as broadcast 5 days after first irrigation. Ten plants were randomly selected in each row of each genotype and tagged to record observations. The observation on disease severity of white rust disease was recorded at 70, 80 and 90 days after sowing (DAS) using 0-9 rating scale (Conn et al, 1990) ${ }^{[4]}$.

$\begin{array}{cc}\text { Rating score } & \text { Leaf area covered }((\%) \\ 0 & \text { No symptoms } \\ 1 & <5 \\ 3 & 5-10 \\ 5 & 11-25 \\ 7 & 26-50 \\ 9 & >50\end{array}$

\section{Disease reaction} Immune (I) Highly resistant (HR) Resistant (R) Moderately resistant (MR) Susceptible (S) Highly susceptible (HS)
With disease observations at different time intervals, Area under disease progress curve (AUDPC) was calculated for each genotypes using formula given by (Wilcox son et al., $1975)^{[22]}$.

$$
A U D P C=\sum_{t=1}^{k-1} \frac{1}{2}\left[\left(x_{i}\right)+\left(x_{i}+1\right) \ldots .\left(x_{i}+n\right)\right]
$$

\section{Where}

$\mathrm{x}_{\mathrm{i}}=$ initial disease index

$x_{i}+1=$ second disease index

The Apparent infection rate was calculated by using formula given by (Vander plank, 1963) ${ }^{[21]}$

$$
r=\frac{2.3}{t_{2}-t_{1}} \log _{10} \frac{x_{2}\left(1-x_{1}\right)}{x_{1}\left(1-x_{2}\right)}
$$




\section{Where}

However, disease response (resistant/susceptible) in all the genotypes was determined at maximum disease pressure at 90 DAS.

2.2 Statistical Analysis: Data obtained from the experiment was analyzed by using OPSTAT software in RBD at $0.05 \%$ probability level.

\section{Result and Discussion}

3.1 Disease reaction on the basis of percent disease severity at 90 DAS

Among different Brassica genotypes evaluated at different time intervals, out of 82 genotypes, 26 genotypes viz. Donskaja, NRCRD 515, Wester, DLSC 1, NBPGR 8, NBPGR 352, NBPGR 407, NBPGR 410, NBPGR 426, NBPGR 427, NBPGR 428, NBPGR 429, NBPGR 433, NBPGR 434, NBPGR 437, NBPGR 439, IC 298024, IC 313379, IC 317528, IC 313378, IC 313379, IC 265495, IC 298024, IC 597942, SIVT 17.108, RTM 16.24, were found immune or free from disease with 0.0 percent disease severity index, while 28 genotypes viz. Candle (18.33), Cutlasss (20.00), Sinapis alba (16.67), Eureca sativa (11.33), NBPGR 1 (25.0), NBPGR 15 (25.0), IC 597932 (20.0), RMWR-09-05 (15.0), DRMRIJ-12-65 (23.3), DRMRIJ-12-06 (25.0), NPJ 217 (15.0), RMM-09-10 (11.67), DRMRIJ-127(21.4), RMWR-09-5-1(11.67), RH-14-001(15.0), DRMR 2035 (11.67), DRMR 2019 (15.0), RLC 5 15.0), DRMR 12.39 (25.0), RL-JEB-52 (15.0), RH 305 (15.0), DRMR-12-48 (11.67), PDZ-5 (11.67), ABS(3)-15 (15.0), RTM-10-9-1 (15.0), 71J0002 (15.0), SIVT 17.19 (11.67), SBZ 13-30 (15.0) were found moderately resistant at 90 DAS. Rest of the genotypes were found susceptible to highly susceptible (28.075.0 percent disease severity) against white rust disease at 90DAS (Table 2). Many earlier workers also evaluated different Brassica genotypes under field conditions and found some genotypes free from disease. Kalpana et al. (2017) [6] reported different Brassica species viz. turnip red (Brassica napus), Sinapis alba, PBC 9221 (Brassica carinata), PBN 9501 (Brassica napus) free from disease while Katili local, Ornamental Rai, Eureca sativa were found resistant against the disease. Based on field studies Saharan and Kaushik,
1988; Gupta et al., (1994) ${ }^{[18,5]}$ reported Brasssica genotypes viz. HC-1, PCC-1 (B. carinata), GSL-1501 (B. napus), EC129126-1 and Shiva free from white rust disease.

Similarly, different B. napus genotypes viz. EC-338997, PBN2001, EC-339000, PBN 2002 and DGS-1 and B. carinata cv. PBC 9221 were free from disease and $B$. juncea genotypes viz. CBJ 001, CBJ 003 and CBJ 004 (China), JM06011 (Australia) were found resistant to white rust disease (Kumar and Kalha, 2005; Li et al., 2008.; Meena et al., 2011) ${ }^{[13,14,15] .}$ In accordance to the present finding this study reveals that Brassica species viz. Sinapis alba and Eureca sativa which were found free from disease and resistant, respectively by Kalpana et al. (2017) ${ }^{[6]}$ reduced their resistance level and found to be moderately resistant against $A$. candida pathogen. Similarly, NBPGR series and IC series which were reported to be immune ( $0 \%$ disease severity) under field conditions were again found free from disease except NBPGR 1, NBPGR 15, IC 597932 which were found moderately resistant with 25.0, 25.0 and 20.0 percent disease severity respectively. Hence continuous screening of resistant genotypes is required to obtain a stable resistant source against the pathogen for effective management (Table 2, figure $1 . \mathrm{A} \& 1 . \mathrm{B})$.

\subsection{Infection rate and area under disease progress curve (AUDPC) in different Brassica genotypes}

Disease severity in different Brassica genotypes at different time intervals (70, 80 \& 90 DAS) clearly revealed gradual progression of disease over time up to 90 DAS. Minimum (0.0949) and maximum (0.1008) infection rate were observed in DRMRJ 127 and 71 J0002 respectively. However, AUDPC for different genotypes was clearly in synchronization with percent disease severity at maximum disease pressure $(90$ DAS) with minimum AUDPC in SIVT-17-19 (69.44) and maximum in RH 30 (588.0). (Table 2, fig. 1 A\& 1 B)). Present finding are in accordance with Kalpana et al (2017) ${ }^{[6]}$ who reported low infection rate and AUDPC in EC 399299 and Raphanus sativus. Kumar et al (2004) ${ }^{[12]}$ also reported resistant genotypes viz. RC 781, RH 819, RC 1425, RH 781 and RH 8119 containing low infection rate and AUDPC in comparison to susceptible genotypes.

Table 2: Disease severity, disease reaction and AUDPC of A. candida on different Brassica species/genotypes under field conditions (Pooled data of 2017-18 and 2018-19)

\begin{tabular}{|c|c|c|c|c|c|c|c|c|c|c|c|c|}
\hline \multirow{2}{*}{ S. No. } & \multirow{2}{*}{ Brassica Genotype } & \multicolumn{9}{|c|}{ \% Disease severity } & \multirow{2}{*}{ Infection Rate } & \multirow[b]{2}{*}{ AUDPC } \\
\hline & & 70DAS & Ang* & DR & 80DAS & Ang* & DR & 90DAS & Ang* & DR & & \\
\hline 1 & Varuna & 40.33 & 39.41 & $\mathrm{~S}$ & 53.33 & 46.89 & HS & 73.33 & 58.91 & HS & 0.0299 & 575.56 \\
\hline 2 & Pusa Bold & 39.33 & 38.82 & $\mathrm{~S}$ & 48.33 & 44.03 & $\mathrm{~S}$ & 65.00 & 53.74 & \begin{tabular}{|l|}
$\mathrm{HS}$ \\
\end{tabular} & 0.0251 & 498.89 \\
\hline 3 & TL 15 & 15.33 & 23.00 & MR & 21.67 & 27.70 & MR & 33.00 & 35.05 & $\mathrm{~S}$ & 0.0383 & 235.56 \\
\hline 4 & Candle & 8.33 & 16.59 & $\mathrm{R}$ & 15.00 & 22.59 & MR & 18.33 & 25.18 & MR & 0.0394 & 138.89 \\
\hline 5 & BSH 1 & 16.00 & 23.56 & MR & 16.67 & 24.04 & MR & 31.67 & 34.22 & $\mathrm{~S}$ & 0.0341 & 207.22 \\
\hline 6 & Kranti & 42.33 & 40.57 & $\mathrm{~S}$ & 51.67 & 45.94 & HS & 65.00 & 53.74 & $\mathrm{HS}$ & 0.0214 & 515.00 \\
\hline 7 & Ragini & 20.00 & 26.55 & MR & 25.00 & 29.98 & MR & 32.00 & 34.43 & $\mathrm{~S}$ & 0.0235 & 245.56 \\
\hline 8 & RL 1359 & 30.00 & 33.20 & $\mathrm{~S}$ & 33.33 & 35.24 & $\mathrm{~S}$ & 53.33 & 46.89 & $\mathrm{HS}$ & 0.0288 & 369.44 \\
\hline 9 & Pusa Kalyani & 22.67 & 28.40 & MR & 21.67 & 27.70 & MR & 36.67 & 37.24 & $\mathrm{~S}$ & 0.0241 & 254.44 \\
\hline 10 & Torch & 16.33 & 23.81 & MR & 15.00 & 22.78 & MR & 30.00 & 33.15 & $S$ & 0.0304 & 193.89 \\
\hline 11 & Tobin & 14.00 & 21.96 & MR & 17.33 & 24.56 & MR & 28.00 & 29.91 & MR & 0.0290 & 187.22 \\
\hline 12 & Bhawani & 22.00 & 27.93 & MR & 32.67 & 34.83 & $\mathrm{~S}$ & 41.67 & 40.18 & $\mathrm{~S}$ & 0.0319 & 315.00 \\
\hline 13 & Sinapis alba & 3.33 & 8.61 & HR & 10.00 & 18.04 & $\mathrm{R}$ & 16.67 & 24.04 & MR & 0.0805 & 113.89 \\
\hline 14 & DLSC 1 & 0.00 & 0.00 & I & 0.00 & 0.00 & I & 0.00 & 0.00 & I & 0.00 & 0.00 \\
\hline 15 & YSPB 24 & 32.33 & 34.63 & $\mathrm{~S}$ & 28.33 & 32.13 & $\mathrm{~S}$ & 43.33 & 41.15 & $\mathrm{~S}$ & 0.0146 & 315.00 \\
\hline 16 & Wester & 0.00 & 0.00 & $\mathrm{I}$ & 0.00 & 0.00 & $\mathrm{I}$ & 0.00 & 0.00 & $\mathrm{I}$ & 0.00 & 0.00 \\
\hline 17 & Heera & 33.33 & 35.24 & $\mathrm{~S}$ & 30.00 & 33.20 & $\mathrm{~S}$ & 43.33 & 41.15 & $\mathrm{~S}$ & 0.0131 & 325.00 \\
\hline 18 & Cutlass & 8.33 & 16.59 & MR & 16.67 & 23.73 & MR & 20.00 & 26.44 & MR & 0.0438 & 155.56 \\
\hline 19 & Sangam & 14.33 & 22.23 & MR & 18.33 & 25.30 & MR & 28.00 & 29.91 & $S$ & 0.0278 & 185.56 \\
\hline
\end{tabular}




\begin{tabular}{|c|c|c|c|c|c|c|c|c|c|c|c|c|}
\hline 20 & RH 30 & 40.00 & 39.22 & $\mathrm{~S}$ & 58.33 & 49.78 & HS & 75.00 & 60.05 & HS & 0.0314 & 588.89 \\
\hline 21 & NRCDR 515 & 0.00 & 0.00 & I & 0.00 & 0.00 & I & 0.00 & 0.00 & I & 0.00 & 0.00 \\
\hline 22 & Eureca sativa & 3.33 & 8.61 & HR & 6.67 & 14.75 & $\mathrm{R}$ & 11.33 & 16.59 & MR & 0.0803 & 91.41 \\
\hline 23 & Donskaja & 0.00 & 0.00 & & 0.00 & 0.00 & I & 0.00 & 0.00 & I & 0.00 & 0.00 \\
\hline 24 & EC 41329 & 36.67 & 37.24 & HS & 41.67 & 40.18 & $\mathrm{~S}$ & 45.00 & 42.10 & $\mathrm{~S}$ & 0.0102 & 391.67 \\
\hline 25 & NBPGR 1 & 4.33 & 11.93 & HR & 11.67 & 19.88 & MR & 25.00 & 29.91 & MR & 0.0876 & 140.56 \\
\hline 26 & NBPGR 8 & 0.00 & 0.00 & I & 0.00 & 0.00 & I & 0.00 & 0.00 & I & 0.00 & 0.00 \\
\hline 27 & NBPGR 15 & 12.33 & 20.49 & MR & 12.33 & 20.49 & MR & 25.00 & 29.91 & MR & 0.0353 & 157.22 \\
\hline 28 & NBPGR 352 & 0.00 & 0.00 & I & 0.00 & 0.00 & I & 0.00 & 0.00 & I & 0.00 & 0.00 \\
\hline 29 & NBPGR 407 & 0.00 & 0.00 & I & 0.00 & 0.00 & I & 0.00 & 0.00 & I & 0.00 & 0.00 \\
\hline 30 & NBPGR 410 & 0.00 & 0.00 & I & 0.00 & 0.00 & I & 0.00 & 0.00 & I & 0.00 & 0.00 \\
\hline 31 & NBPGR 426 & 0.00 & 0.00 & I & 0.00 & 0.00 & I & 0.00 & 0.00 & I & 0.00 & 0.00 \\
\hline 32 & NBPGR 427 & 0.00 & 0.00 & I & 0.00 & 0.00 & I & 0.00 & 0.00 & I & 0.00 & 0.00 \\
\hline 33 & NBPGR 428 & 0.00 & 0.00 & I & 0.00 & 0.00 & I & 0.00 & 0.00 & I & 0.00 & 0.00 \\
\hline 34 & NBPGR 429 & 0.00 & 0.00 & I & 0.00 & 0.00 & I & 0.00 & 0.00 & I & 0.00 & 0.00 \\
\hline 35 & NBPGR 433 & 0.00 & 0.00 & I & 0.00 & 0.00 & I & 0.00 & 0.00 & I & 0.00 & 0.00 \\
\hline 36 & NBPGR 434 & 0.00 & 0.00 & I & 0.00 & 0.00 & I & 0.00 & 0.00 & I & 0.00 & 0.00 \\
\hline 37 & NBPGR 437 & 0.00 & 0.00 & I & 0.00 & 0.00 & I & 0.00 & 0.00 & I & 0.00 & 0.00 \\
\hline 38 & NBPGR 439 & 0.00 & 0.00 & I & 0.00 & 0.00 & I & 0.00 & 0.00 & I & 0.00 & 0.00 \\
\hline 39 & PRD.14.16 & 42.00 & 40.38 & $\mathrm{~S}$ & 53.33 & 46.89 & $\mathrm{HS}$ & 66.67 & 54.76 & HS & 0.0231 & 497.78 \\
\hline 40 & PRD.14.25 & 38.33 & 38.23 & $\mathrm{~S}$ & 38.33 & 38.23 & $\mathrm{~S}$ & 45.00 & 42.10 & $\mathrm{~S}$ & 0.0080 & 372.22 \\
\hline 41 & PRD.34.20 & 51.67 & 45.94 & HS & 53.33 & 46.89 & HS & 66.67 & 54.76 & HS & 0.0127 & 530.56 \\
\hline 42 & PAB.14.57 & 40.00 & 39.20 & $\mathrm{~S}$ & 41.67 & 40.18 & $\mathrm{~S}$ & 45.00 & 42.10 & $\mathrm{~S}$ & 0.0059 & 391.67 \\
\hline 43 & IC 313379 & 0.00 & 0.00 & I & 0.00 & 0.00 & I & 0.00 & 0.00 & I & 0.00 & 0.00 \\
\hline 44 & IC 298024 & 0.00 & 0.00 & I & 0.00 & 0.00 & I & 0.00 & 0.00 & I & 0.00 & 0.00 \\
\hline 45 & IC 597932 & 6.67 & 13.70 & $\mathrm{R}$ & 12.67 & 20.81 & MR & 20.00 & 27.22 & MR & 0.0240 & 126.06 \\
\hline 46 & IC 317528 & 0.00 & 0.00 & I & 0.00 & 0.00 & I & 0.00 & 0.00 & I & 0.00 & 0.00 \\
\hline 47 & IC 313378 & 0.00 & 0.00 & I & 0.00 & 0.00 & I & 0.00 & 0.00 & I & 0.00 & 0.00 \\
\hline 48 & IC 313379 & 0.00 & 0.00 & I & 0.00 & 0.00 & I & 0.00 & 0.00 & I & 0.00 & 0.00 \\
\hline 49 & IC 265495 & 0.00 & 0.00 & I & 0.00 & 0.00 & I & 0.00 & 0.00 & I & 0.00 & 0.00 \\
\hline 50 & IC 298024 & 0.00 & 0.00 & I & 0.00 & 0.00 & I & 0.00 & 0.00 & I & 0.00 & 0.00 \\
\hline 51 & IC 597942 & 0.00 & 0.00 & I & 0.00 & 0.00 & I & 0.00 & 0.00 & I & 0.00 & 0.00 \\
\hline 52 & RMWR-09-05 & 3.00 & 9.72 & HR & 5.00 & 12.92 & HR & 15.00 & 22.59 & MR & 0.0805 & 76.67 \\
\hline 53 & DRMRIJ-12-65 & 10.00 & 18.43 & $\mathrm{R}$ & 11.67 & 19.88 & MR & 23.33 & 28.65 & MR & 0.0424 & 144.44 \\
\hline 54 & DRMRIJ-12-43 & 20.00 & 26.55 & MR & 30.00 & 33.15 & $\mathrm{~S}$ & 41.67 & 40.18 & $\mathrm{~S}$ & 0.0367 & 300.00 \\
\hline 55 & DRMRIJ-12-02 & 18.33 & 25.30 & MR & 21.67 & 27.70 & MR & 36.67 & 37.19 & $\mathrm{~S}$ & 0.0347 & 252.78 \\
\hline 56 & RH 1231 & 26.67 & 31.06 & $\mathrm{~S}$ & 31.67 & 34.22 & $\mathrm{~S}$ & 38.33 & 38.23 & $S$ & 0.0181 & 313.89 \\
\hline 57 & DRMRIJ 12-06 & 12.33 & 20.49 & MR & 15.67 & 23.20 & MR & 25.00 & 29.91 & MR & 0.0353 & 170.56 \\
\hline 58 & NPJ 217 & 4.33 & 11.93 & HR & 6.67 & 14.75 & $\mathrm{R}$ & 15.00 & 22.59 & MR & 0.0621 & 82.78 \\
\hline 59 & EC 399301 & 26.67 & 31.06 & $\mathrm{~S}$ & 35.00 & 36.22 & $\mathrm{~S}$ & 50.00 & 44.98 & $\mathrm{~S}$ & 0.0178 & 335.56 \\
\hline 60 & RMM-09-10 & 3.00 & 9.72 & HR & 6.67 & 14.75 & $\mathrm{R}$ & 11.67 & 19.88 & MR & 0.0679 & 72.22 \\
\hline 61 & DRMRJ 127 & 2.00 & 8.13 & HR & 10.00 & 18.04 & $\mathrm{R}$ & 13.33 & 21.14 & MR & 0.0949 & 87.22 \\
\hline 62 & RMWR-09-5-1 & 5.00 & 12.92 & HR & 6.67 & 14.75 & $\bar{R}$ & 11.67 & 19.88 & MR & 0.0424 & 74.44 \\
\hline 63 & RH.14.001 & 6.67 & 14.75 & $\mathrm{R}$ & 6.67 & 14.75 & $\mathrm{R}$ & 15.00 & 22.59 & MR & 0.0406 & 88.33 \\
\hline 64 & DRMR 2035 & 6.67 & 14.75 & $\mathrm{R}$ & 6.67 & 14.75 & $\mathrm{R}$ & 11.67 & 19.88 & MR & 0.0280 & 77.22 \\
\hline 65 & DRMR 2019 & 5.00 & 12.92 & HR & 7.33 & 15.59 & $\mathrm{R}$ & 15.00 & 22.59 & MR & 0.0549 & 87.78 \\
\hline 66 & RLC-5 & 4.00 & 11.32 & HR & 6.67 & 14.75 & $\mathrm{R}$ & 15.00 & 22.59 & MR & 0.0661 & 82.22 \\
\hline 67 & DRMR 12.39 & 8.33 & 16.59 & $\mathrm{R}$ & 13.00 & 21.10 & MR & 25.00 & 29.91 & MR & 0.0549 & 151.67 \\
\hline 68 & DRMRIJ 12.40 & 18.33 & 25.30 & MR & 26.67 & 31.06 & $\mathrm{~S}$ & 45.33 & 42.30 & $\mathrm{~S}$ & 0.0453 & 292.78 \\
\hline 69 & RL-JEB-52 & 5.00 & 12.92 & HR & 6.67 & 14.75 & $\mathrm{R}$ & 15.00 & 22.59 & MR & 0.0549 & 85.56 \\
\hline 70 & RH 305 & 4.00 & 11.32 & HR & 10.33 & 18.46 & $\mathrm{R}$ & 15.00 & 22.59 & MR & 0.0661 & 99.44 \\
\hline 71 & DRMR-12-48 & 5.00 & 12.92 & HR & 8.33 & 16.59 & $\mathrm{R}$ & 11.67 & 19.88 & MR & 0.0168 & 74.44 \\
\hline 72 & RMM-09-6-1 & 35.00 & 36.22 & $\mathrm{~S}$ & 50.00 & 44.98 & $\mathrm{~S}$ & 55.00 & 47.86 & HS & 0.0226 & 452.78 \\
\hline 73 & PDZ 5 & 6.67 & 14.75 & $\mathrm{R}$ & 10.00 & 18.04 & $\mathrm{R}$ & 11.67 & 19.88 & MR & 0.0280 & 85.56 \\
\hline 74 & $\operatorname{ABS}(3)-15$ & 6.67 & 14.75 & $\mathrm{R}$ & 8.33 & 16.59 & $\mathrm{R}$ & 15.00 & 22.59 & MR & 0.0294 & 92.22 \\
\hline 75 & RTM-10-9-1 & 3.00 & 9.72 & HR & 8.33 & 16.59 & $\mathrm{R}$ & 15.00 & 22.59 & MR & 0.0805 & 91.11 \\
\hline 76 & PDZ 3 & 15.00 & 22.59 & MR & 23.33 & 28.84 & MR & 35.00 & 36.22 & $\mathrm{~S}$ & 0.0424 & 241.11 \\
\hline 77 & DRMR 5206 & 16.67 & 24.04 & MR & 36.67 & 37.24 & $\mathrm{~S}$ & 35.00 & 36.22 & $S$ & 0.0371 & 305.56 \\
\hline 78 & $71 \mathrm{~J} 0002$ & 2.00 & 8.13 & HR & 9.67 & 17.91 & $\mathrm{R}$ & 15.00 & 22.59 & MR & 0.1008 & 91.67 \\
\hline 79 & RTM 16.24 & 0.00 & 0.00 & I & 0.00 & 0.00 & I & 0.00 & 0.00 & I & 0.00 & 0.00 \\
\hline 80 & SIVT 17.108 & 0.00 & 0.00 & I & 0.00 & 0.00 & I & 0.00 & 0.00 & I & 0.00 & 0.00 \\
\hline 81 & SIVT 17.19 & 2.00 & 8.13 & HR & 6.67 & 14.75 & $\mathrm{R}$ & 11.67 & 19.88 & MR & 0.0882 & 69.44 \\
\hline 82 & SBZ 1330 & 6.67 & 14.75 & $\mathrm{R}$ & 13.33 & 20.95 & MR & 15.00 & 22.59 & MR & 0.0406 & 107.78 \\
\hline \multicolumn{2}{|r|}{ CD 5\% } & 3.49 & & & 3.68 & & & 4.37 & & & & \\
\hline \multicolumn{2}{|r|}{$\mathrm{CV}$} & 11.18 & & & 10.31 & & & 9.76 & & & & \\
\hline
\end{tabular}

I: Immune, HR: Highly resistant, MR: Moderately resistant, R: Resistant,

S: Susceptible, HS: Highly susceptible 


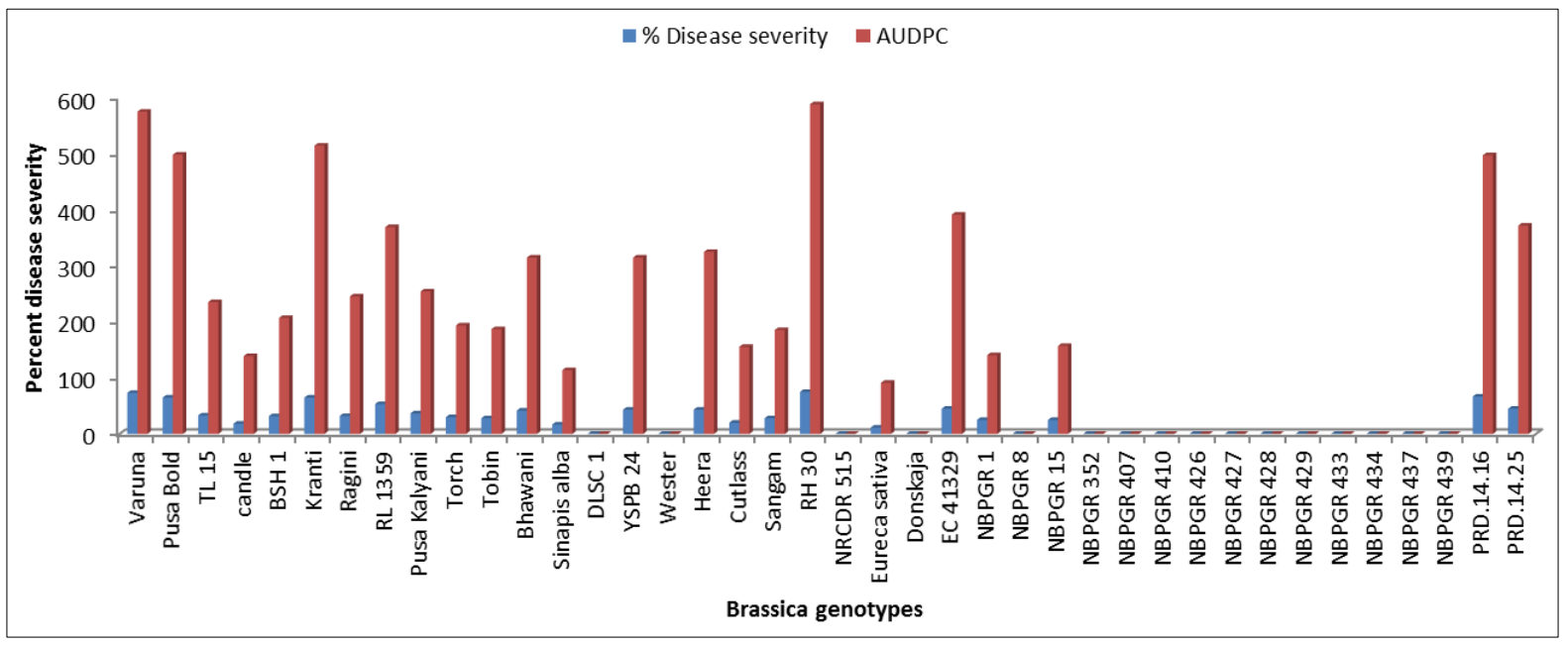

Fig 1(A): Percent disease severity (90DAS) and AUDPC of A. candida on different Brassica species under field conditions

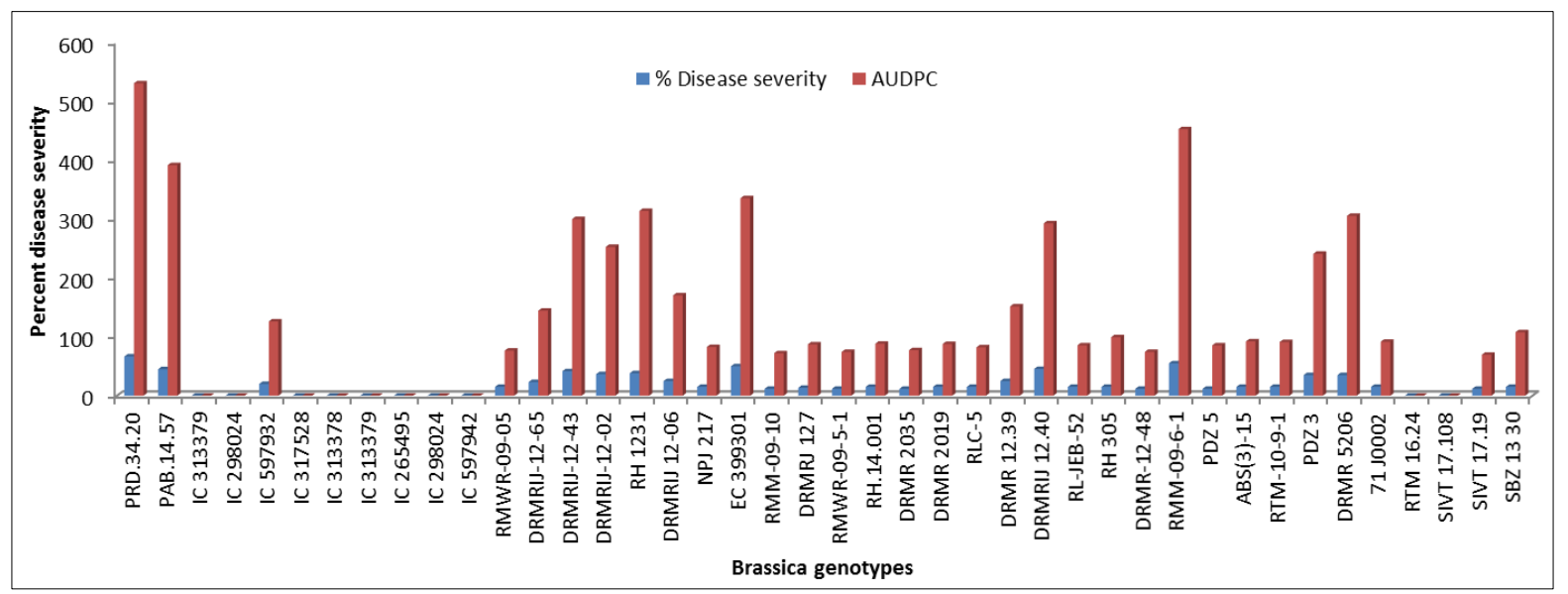

Fig 1(B): Percent disease severity (90DAS) and AUDPC of A. candida on different Brassica genotypes under field conditions

\section{Conclusion}

The present investigation on evaluation of different Brassica genotypes under natural field conditions against white rust disease revealed that continuous evaluation of new genotypes as well as existing resistant genotypes is needed as evolution and emergence of new pathogenic races, may cause break down of existing resistant sources. However, for the final confirmation of resistance, the genotypes which have been found free from disease or resistant under field conditions need to be evaluated in glasshouse also under artificial epiphytotic conditions to assure if absence of disease in field condition is due to true resistance in that genotype or an escape from disease due to unfavorable environmental conditions for disease development. The Brassica species which are free from disease or highly resistant against $A$. candida pathogen can not only be used as resistant sources against the disease but also as potential sources for selection of differential hosts for finding out $A$. candida pathotypes/races.

\section{Acknowledgment}

I gratefully acknowledge Oilseed lab, Plant Pathology Dept., GBPUA\&T, Pantnagar for proving support and entries which greatly assisted the research.

\section{References}

1. Anonymous 2020. http://www.drmr.res.in/seed_plant.php

2. Ashrafuzzaman M, Sarkar MEH, Rahman GMM, Islam T. Development of Alternaria blight (Alternaria brassicae) in mustard and its relation to yield and yield contributed characters. Bangladesh Journal of Plant Pathology 1996;12:1-4.

3. Bisht IS, Agrawal RC, Singh R. White rust severity in mustard varieties and its effect on seed yield. Plant Varieties and Yield 1994;7:85-89.

4. Conn KL, Tewari JP, Awasthi RP. A disease assessment key for Alternaria black spot in rapeseed and mustard. Canadian Plant Disease Survey 1990;70:19-22.

5. Gupta RBL, Singh M. Source of resistance to white rust and powdery mildew of mustard. International Journal of Tropical Plant Disease 1994;12:225-227.

6. Kalpana Gairola, Tewari AK. Evaluation of Brassica germplasm for resistance sources against white rust. International journal of Environment, Agriculture and Biotechnology 2017;2(3):1215-1226.

7. Kalpana Gairola, Tewari AK. Management of white rust (Albugo candida) in Indian mustard by fungicides and garlic extract. Pesticide Research Journal 2019;31(1):6065.

8. Kolte SJ. Diseases. In: Oilseeds and vegetable Brassicas. An Indian perspective (eds. Chopra, V.L. and Prakash S.). Oxford and IBH Publishing Co. Pvt. Ltd. New Delhi 1996, 184-207.

9. Kolte SJ, Tewari AN. Note on the susceptibility of the certain oliferous Brassicae to downy mildew and white blister disease. Indian Journal of Mycology and Plant Pathology 1980;10:191-192. 
10. Kolte SJ, Sharma KD, Awasthi RP. Yield losses and control of downy mildew and white rust of rapeseed and mustard. In: $3^{\text {rd }}$ international symposium plant pathology. December, 14-18, IARI, New Delhi, India Session 1981;12:70-71.

11. Kumar B, Kolte SJ. Progression of Alternaria blight of mustard in relation to component of resistance. Indian Phytopathology 2001;3:329-331.

12. Kumar, Birendra, Dang JK, Sharma OP. Component of Partial resistant to Albugo candida in Rapeseed mustard. Journal of Mycology and Plant Pathology 2004;2:645649.

13. Kumar, Sudhir, Kalha CS. Evaluation of rapeseed mustard germplasm against white rust and Alternaria blight. Annals of Biology 2005;1:73-163.

14. Li CX, Sivasithamparam K, Walton G, Fels P, Barbetti MJ. Both incidence and severity of white rust disease reflect host resistance in Brassica juncea germplasm from Australia, China and India. Field Crop Research 2008;108:1-8.

15. Meena PD, Chattopadhyay C, Kumar A, Awasthi RP, Singh R, Kaur S et al. Comparative study on effect of chemicals on Alternaria blight in Indian mustard-A multi location study in Indian. Journal of Environmental Biology 2011;32:375-379.

16. Panjabi-Massand P, Yadava SK, Sharma P, Kaur A, Kumar A, Arumugam $\mathrm{N}$ et al. Molecular mapping reveals two independent loci conferring resistance to Albugo candida in the east European germplasm of oilseed mustard Brassica juncea. Theor. Appl. Genet 2010;121(1):137-145.

17. Saharan GS, Verma PR. White Rusts: A review of economically important species. International Development Research Center (IDRC), Ottawa. Canada MR 315e 1992, 65.

18. Saharan GS, Kaushik CD, Kaushik JC. Sources of resistance and epidemiology of white rust of mustard. Indian Phytopathology 1988;41:96-99.

19. Saharan GS. Management of rapeseed and mustard diseases. In: Advances in oilseed research (D. Kumar \& M. Rai eds.) Jodhpur, Sci. Pub 1992b, 151-188.

20. Saharan GS, Kaushik CD, Gupta PP, Tripathi NN. Assessment of losses and control of white rust of mustard. Indian phytopathology 1984;37:397.

21. Vanderplank JE. Plant disease: Epidemics and Control. Academic Press, New York 1963, 349.

22. Wilcoxson RD, Skovmand B, Atif AH. Evaluation of wheat cultivars for ability to retard development of stem rust. Annual Applied Biology 1975;80:275-281. 